\title{
Estudio y seguimiento cardiovascular de niños y adolescentes sometidos a tratamientos oncohematológicos. ¿Por qué, cómo y cuándo?
}

\section{Study and cardiovascular follow-up of children and adolescents undergoing oncohematological treatments. Why, how and when?}

María G. Jiménez-Carbajal*

Cardiología Intervencionista Pediátrica/Ecocardiografía Pediátrica, Centro Médico ABC, Ciudad de México, México

\begin{abstract}
Resumen
Estudio de revisión de diversas publicaciones internacionales en la literatura médica relacionados con la evaluación cardiovascular en pacientes en edad pediátrica sometidos a algún tipo de tratamiento oncohematológico, previendo factores de riesgo que en conjunto con la quimioterapia y/o radioterapia merman el adecuado funcionamiento cardiovascular de forma particular.
\end{abstract}

Palabras clave: Cardiotoxicidad. Tratamientos oncohematológicos. Niños y adolescentes.

\begin{abstract}
Review of several international publications in the medical literature related to cardiovascular evaluation in pediatric patients undergoing some type of onco-hematological treatment, providing risk factors that together with chemo and/or radiotherapy, impair adequate cardiovascular function in a particular way.
\end{abstract}

Key words: Cardiotoxicity. Onco-hematological treatments. Children and adolescents.

\section{Introducción}

Debido al importante incremento del diagnóstico y tratamiento, a base de quimioterapia y radioterapia, de padecimientos oncohematológicos en pacientes en edad pediátrica y bajo el conocimiento de que las complicaciones cardiovasculares son la segunda causa de morbilidad y mortalidad en pacientes supervivientes, es necesario conocer y difundir los fundamentos teóricos de los efectos de las terapéuticas empleadas para tal fin, así como las bases para la detección y manejo precoces de eventos adversos que se relacionen con ellas.

\section{Método}

Este es un estudio de revisión internacional de datos publicados motivado por la observación de las complicaciones ocurridas en pacientes con padecimientos

\section{Correspondencia:}

*María G. Jiménez-Carbajal

E-mail: mgjcmed@yahoo.com.mx,

unicardin@gmail.com
Fecha de recepción: 18-07-2019

Fecha de aceptación: 04-05-2020 DOI: 10.24875/ACM.20000297
Disponible en internet: 03-08-2020 Arch Cardiol Mex. 2020;90(4):511-519 www.archivoscardiologia.com 1405-9940 / @ 2020 Instituto Nacional de Cardiología Ignacio Chávez. Publicado por Permanyer. Este es un artículo open access bajo la licencia CC BY-NC-ND (http://creativecommons.org/licenses/by-nc-nd/4.0/). 
oncohematológicos, tanto durante la administración de los medicamentos como en aquellos que ya se encuentran en etapa de supervivencia, con el incremento del riesgo cardiovascular inherente a los factores poblacionales modificables y no modificables por todos conocidos.

\section{Resultados}

\section{Epidemiología}

Actualmente se estima que existe una tasa de curación superior al $80 \%$ de niños con padecimientos oncohematológicos, lo que aumenta la expectativa de vida libre de enfermedad, pero incrementa la toxicidad multiorgánica, incluyendo la cardiaca, lo que genera la necesidad de detección y manejo temprano de las complicaciones, con la finalidad de mejorar la calidad de vida de los pacientes ${ }^{1-3}$. Cerca del $60 \%$ de los pacientes en edad pediátrica con enfermedades oncohematológicas reciben antracíclicos ${ }^{3}$. Se estima que hasta el $65 \%$ de los sobrevivientes de cáncer infantil tratados con antraciclinas podrían cursar con disfunción miocárdica subclínica ${ }^{4}$; en este grupo de pacientes la enfermedad cardiovascular es la principal causa de morbilidad y mortalidad por cardiotoxicidad, después de la recurrencia del cáncer y malignidades secundarias $^{1,5-7}$. La incidencia de insuficiencia cardiaca clínica se ha reportado hasta del 16\%, 0.9 a 4.8 años después de la quimioterapia ${ }^{1,5}$.

\section{Cardiotoxicidad}

Son las alteraciones cardiovasculares derivadas de los tratamientos oncohematológicos ${ }^{8}$. Se puede definir con base en el deterioro de la fracción de eyección del ventrículo izquierdo (FEVI) como grado I: reducción de la FEVI del 10 al $20 \%$ con respecto a la basal; grado II: reducción $>20 \%$ o caída por debajo de lo normal, y grado III: aparición de síntomas de insuficiencia cardiaca congestiva ${ }^{9,10}$. La Sociedad Americana de Ecocardiografía y la Asociación Europea de Imagen Cardiovascular utilizan el 53\% como límite normal de $\mathrm{FEVI} \mathbf{8}^{8,11}$.

\section{Factores de riesgo}

\section{Modificables}

- Factores relacionados con la terapia como la dosis acumulada de antraciclinas, dosis totales administradas en un día o ciclo y la administración concomitante con otros cardiotóxicos. Existen reportes de disfunción de ventrículo izquierdo $(\mathrm{VI})$ a dosis tan bajas como $240 \mathrm{mg} / \mathrm{m}^{2}$, por lo que algunos autores consideran que no hay "dosis segura" del fármaco $0^{1,12,13}$. La radiación mediastínica con o sin tratamiento con antraciclina es otro factor de riesgo de cardiotoxicidad, presentándose en los casos de radiación cardiaca aún con bajas dosis ${ }^{14-16}$. La radiación craneal es también un factor de riesgo para las enfermedades cardiovasculares y anomalías probablemente mediada por la deficiencia de la hormona del crecimiento y relacionadas con menor masa del VI ${ }^{17-19}$.

- Los factores individuales incluyen sedentarismo, trastornos electrolíticos (principalmente hipopotasemia e hipomagnesemia), hipertensión, dislipidemia, endocrinopatías, obesidad, diabetes, consumo de drogas o de alcohol y tabaquismo ${ }^{1,12}$.

\section{No MODIFICABLES}

Los tumores en la edad pediátrica pueden tener periodos de latencia más bajos, crecer más rápidamente y volverse muy invasivos, la mayoría presentan tipos histológicos que se asemejan a los tejidos fetales en diferentes etapas de desarrollo, considerándose embrionarios, lo que genera una gran diversidad morfológica con grados variados de diferenciación celular $^{12}$. La edad $<5$ años en el momento del inicio del tratamiento representa un importante factor de riesgo para cardiotoxicidad con incremento de la poscarga y disminución de la masa y grosor de las paredes del $\mathrm{VI}^{1,12,20,21}$. Los pacientes de sexo femenino tienen un mayor porcentaje de grasa corporal y la doxorubicina es poco soluble en grasas, por lo que el mayor riesgo entre las niñas puede relacionarse con concentraciones de doxorubicina más bajas ${ }^{22,23}$. Los niños con enfermedades cardiovasculares preexistentes, los niños con cáncer y concentraciones elevadas de troponina pretratamiento, algunos tipos genéticos tumorales (mutación HFE C282Y), así como pacientes con cromosomopatías como la trisomía 21 tienen un mayor riesgo de cardiotoxicidad relacionada con las antraciclinas ${ }^{13,24-26}$.

\section{Clasificación}

La cardiotoxicidad se puede presentar durante el tratamiento y hasta 40 años después de finalizada la terapia, clasificándose en aguda o subaguda cuando se desarrolla desde el inicio del tratamiento, hasta dos semanas después de terminado ${ }^{27,28}$ y crónica cuando 
Tabla 1. Fármacos usados en oncohematología pediátrica, potencialmente cardiotóxicos

\begin{tabular}{|c|c|c|}
\hline Fármaco & Usos en oncohematología pediátrica & Efectos cardiovasculares \\
\hline $\begin{array}{l}\text { Antracíclicos (doxorubicina, daunorubicina, } \\
\text { idarubicina y/o epirubicina) }\end{array}$ & $\begin{array}{l}\text { LLA, diversos tumores sólidos como } \\
\text { osteosarcoma }\end{array}$ & $\begin{array}{l}\mathrm{CMD} \text {, CMR, arritmias, prolongación del intervalo } \\
\mathrm{OT} \text {, disfunción del nodo sinusal, IC }\end{array}$ \\
\hline Bleomicina & $\begin{array}{l}\text { LH, tumores de células germinales, } \\
\text { carcinomas }\end{array}$ & Isquemia, pericarditis, fibrosis pulmonar \\
\hline Ciclofosfamida, ifosfamida (en combinación) & LNH, leucemias, LH, linfoma de Burkitt & $\begin{array}{l}\text { Cardiotoxicidad aguda, desde arritmias sutiles } \\
\text { hasta CMD fatal. Miocarditis, IC }\end{array}$ \\
\hline 5-Fluoruracilo & $\begin{array}{l}\text { Hepatoblastoma, carcinoma } \\
\text { rinofaríngeo, tumores de células } \\
\text { germinales }\end{array}$ & Arritmias, isquemia, IC y muerte súbita \\
\hline Inhibidores multicinasa, sutinib y sorafenib & LMC & $\begin{array}{l}\text { Angor pectoris, IC, alteraciones } \\
\text { electrocardiográficas }\end{array}$ \\
\hline Vincristina, vinblastina & $\begin{array}{l}\text { LLA, linfomas, tumor de Wilms, } \\
\text { sarcoma de Ewing, neuroblastoma y } \\
\text { rabdomiosarcoma }\end{array}$ & $\begin{array}{l}\text { HTA, isquemia miocárdica, complicaciones } \\
\text { venooclusivas }\end{array}$ \\
\hline Citarabina & Algunos tipos de leucemia & $\begin{array}{l}\text { Pericarditis, derrame pericárdico, taponamiento } \\
\text { cardiaco }\end{array}$ \\
\hline Cisplatino, carboplatino & Tumores cerebrales, osteosarcoma & $\begin{array}{l}\text { FA, TSV, bloqueo de rama izquierda e infarto del } \\
\text { miocardio }\end{array}$ \\
\hline Bevacizumab & $\begin{array}{l}\text { Cáncer de pulmón, de ovario, de } \\
\text { trompas de Falopio, peritoneal } \\
\text { primario }\end{array}$ & HTA, TEA, IC, isquemia, TSV \\
\hline Irinotecán & $\begin{array}{l}\text { Meduloblastoma, tumores } \\
\text { neuroectodérmicos primitivos, } \\
\text { rabdomiosarcoma y neuroblastoma }\end{array}$ & Bradicardia, hipotensión \\
\hline Esteroides & LLA & HTA, prolongación de intervalo $\mathrm{QT}$ \\
\hline
\end{tabular}

LLA: leucemia linfoblástica aguda; CMD: cardiomiopatía dilatada; CMR: cardiomiopatía restrictiva; IC: insuficiencia cardiaca; LLR: leucemia linfoblástica refractaria; VI: ventrículo izquierdo; LNH: linfoma no Hodgkin; LH: linfoma de Hodgkin; LMC: leucemia mieloide crónica; HTA: hipertensión arterial; TEA: tromboembolia arterial; FA: fibrilación auricular; TSV: taquicardia supraventricular.

la toxicidad aparece posterior a un año de completar la terapia. Esta última, a su vez, se divide en temprana cuando ocurre en el primer año posterior a la terapia y tardía si ocurre años después de finalizarla ${ }^{10,29-31}$.

\section{Mecanismo de cardiotoxicidad}

El objetivo de la quimioterapia es la inhibición de la división celular, sin embargo, su efecto clínico no es selectivo, afecta a las células no cancerígenas propias del organismo y genera toxicidad ${ }^{27,32}$. Los antineoplásicos que tienen el riesgo de producir cardiotoxicidad se clasifican en dos tipos ${ }^{27}$ :

- Tipo I: cardiotoxicidad por antraciclinas. Su toxicidad cardiaca es dependiente de la dosis y produce daño cardiaco irreversible ${ }^{27}$. Ningún paciente que recibe antraciclinas está exento de daño cardiaco, aunque sea mínimo ${ }^{3}$. Su presentación puede ser aguda (independiente de la dosis y transitoria relacionada con la hipersensibilidad tipo 1) o crónica (dependiente de la dosis acumulativa, asociada a un aumento en la apoptosis) ${ }^{27,33-35}$. La cardiomiopatía dilatada podría progresar hasta una cardiomiopatía restrictiva, por disminución del número de cardiomiocitos funcionales y de células madre capaces de regenerar el tejido cardiaco $0^{4,20,36-38}$.

- Tipo Il: cardiotoxicidad por trastuzumab. Causa daño cardiaco reversible que permite una recuperación de la funcionalidad y un reinicio del régimen si está indicado ${ }^{12,27,39}$.

La terapia combinada con agentes tipo I y II se asocia con mayor incidencia de cardiotoxicidad $(27 \%)^{27,33,40}$. Existen otros fármacos cardiotóxicos. En la tabla 1 se describen algunos de los fármacos más utilizados en oncología y hematología pediátrica, citando sus efectos cardiovasculares $4,8,9,12,20,36-38,40$. 


\section{Mecanismo de daño de la radiación}

La radioterapia puede causar efectos secundarios cardiotóxicos como fibrosis miocárdica, cardiomiopatía, enfermedad coronaria precoz, disfunción valvular y electrofisiológica. Esta cardiotoxicidad es dependiente de la dosis y se correlaciona con el área del corazón expuesta, la técnica radiológica y la edad del paciente, con mayor incidencia en los pacientes más jóvenes. Está causada por daño agudo y por inflamación. Los pacientes que reciben más de 1,500-3,500 cGy muestran un riesgo de dos a seis veces mayor de enfermedad cardiaca; relacionándose las altas dosis con isquemia miocárdica desde los 12 años posteriores al tratamiento ${ }^{34,41-43}$.

\section{Diagnóstico}

Todos los pacientes que serán tratados con fármacos oncológicos potencialmente cardiotóxicos deben tener un examen clínico minucioso en el que se evalúe la presencia de antecedentes cardiovasculares como cardiopatías congénitas, valvulopatías, miocardiopatías, antecedente de disfunción de VI aún asintomática, antecedente de tratamiento previo con antineoplásicos o la irradiación torácica previa. Se recomienda la monitorización frecuente de los signos vitales durante la infusión de los agentes quimioterapéuticos ${ }^{9}$.

\section{ELECTROCARDIOGRAMA}

Se recomienda en todos los pacientes antes y durante el tratamiento. Se debe detectar cualquier signo electrocardiográfico de toxicidad cardiaca como taquicardia en reposo, cambios del intervalo ST, trastornos de la conducción, prolongación del intervalo QT o arritmias. Estas alteraciones no son específicas, pueden ser transitorias y pueden no relacionarse con el desarrollo de cardiomiopatía crónica ${ }^{44}$.

\section{ECOCARDIOGRAMA}

Se debe obtener una evaluación basal de la FEVI al inicio del tratamiento para la comparación con estudios posteriores. Una $\mathrm{FEVI}<53 \%$ es un factor de riesgo para el desarrollo de insuficiencia cardiaca, si esta es $\leq 50 \%$ no se recomienda iniciar un esquema de tratamiento con fármacos de alto potencial cardiotóxico, debiendo evaluarse en conjunto con el oncólogo la posibilidad de un esquema alternativo. Si bien la FEVI es el parámetro universalmente utilizado para la toma de decisiones, no es sensible para la detección precoz del compromiso cardiaco subclínico y depende de los cambios de precarga y poscarga que conducen a cambios transitorios en ella. Se ha observado que la determinación de las velocidades miocárdicas con Doppler tisular a nivel del anillo mitral puede ser más sensible para la detección de disfunción ventricular incipiente ${ }^{9,45}$. El Doppler pulsado del tracto de entrada del VI permite evaluar la presencia de un patrón restrictivo, la relación E/E' aumentada (> 15) es un parámetro específico de aumento de la presión telediastólica y se asocia con el desarrollo de insuficiencia cardiaca9,46. La determinación de la deformación y de la velocidad de deformación (strain y strain rate) actualmente juegan un papel preponderante en la detección precoz de disfunción ventricular ${ }^{9,47-49}$. En situación basal, el strain longitudinal global (SLG) mejora la estratificación del riesgo de disfunción ventricular por cardiotoxicidad frente a la FEVI-2D. Durante el tratamiento antitumoral, el SLG detecta daño miocárdico precozmente y con menos variabilidad que la FEVI. El uso combinado de SLG y troponina I mejora el valor predictivo negativo para disfunción ventricular ${ }^{8}$. El método actualmente preferido para la estimación de la FEVI es mediante ecocardiografía tridimensional, la cual tiene menos variabilidad con respecto al método bidimensional ${ }^{8}$. Deberá realizarse siempre que se cuente con dicho recurso.

\section{RESONANCIA NUCLEAR MAGNÉTICA}

Método de elección para la evaluación de la masa cardiaca, los volúmenes y la fracción de eyección. Se usa principalmente cuando las imágenes de ecocardiografía son subóptimas o cuando hay discrepancia en la medición de la FEV|27.

\section{Medicina nUCLEAR}

Tiene alta reproducibilidad en el cálculo de la FEVI con baja variabilidad interobservador. Como desventajas están la exposición a radiación ionizante y la imposibilidad de valorar las válvulas y el pericardio ${ }^{27}$.

\section{Biomarcadores}

Los biomarcadores plasmáticos específicos de lesión miocárdica son una herramienta útil para la detección y la evaluación precoz de la cardiotoxicidad ${ }^{50}$. 


\section{Troponinas}

Son marcadores de lesión miocárdica por excelencia ${ }^{51}$. En pacientes que han recibido diversos quimioterápicos se han detectado niveles elevados tanto de troponina $T$ como de troponina I al ser determinados precozmente en relación con el momento de recibir el tratamiento antineoplásico. Esto se correlaciona con mayor incidencia y gravedad de disfunción del VI en el seguimiento ${ }^{52-54}$. En pacientes que reciben trastuzumab y muestran elevaciones de la troponina I existe una posibilidad mayor de que presenten disfunción ventricular y que tengan una probabilidad menor de recuperación con respecto a aquellos pacientes con niveles normales de troponina ${ }^{55}$.

\section{Péptidos natriuréticos}

El péptido natriurético cerebral (BNP) y la fracción amino terminal de propéptido natriurético cerebral (NT-proBNP) son biomarcadores de valor pronóstico y posible guía del tratamiento de los pacientes en este contexto ${ }^{53,56}$. En el ámbito de la disfunción ventricular izquierda y la insuficiencia cardiaca asociadas con cardiotoxicidad por quimioterápicos hay suficiente evidencia de que elevaciones persistentes de estos marcadores se asocian con mayor riesgo de desarrollo de disfunción ventricular (tanto sistólica como diastólica) ${ }^{57-59}$.

\section{BIOPSIA ENDOMIOCÁRDICA}

Es el método más fidedigno para evaluar la lesión miocárdica, no obstante, al ser invasiva, obtener pequeñas muestras del miocardio y no aportar información funcional se ha limitado su uso en el monitoreo y el diagnóstico de la cardiotoxicidad ${ }^{27}$.

\section{Prevención de la cardiotoxicidad}

Es obligado promover un estilo de vida cardiosaludable con programas de ejercicio físico regular, para todos los pacientes, independientemente del tratamiento planeado ${ }^{8}$. Se recomienda no exceder la dosis equivalente a $375 \mathrm{mg} / \mathrm{m}^{2}$ de doxorubicina en niños, umbral que se deberá reducir a $300 \mathrm{mg} / \mathrm{m}^{2}$ en el caso de radiación asociada en área cardiaca ${ }^{60}$. Se han propuesto las infusiones prolongadas de antraciclinas (> 6 y hasta $48 \mathrm{~h}$ ), en lugar de bolos; sin embargo, algunos autores proponen que la exposición prolongada de los cardiomiocitos al antracíclico pudiera ocasionar mayor daño cardiaco. Este abordaje tiene la desventaja de comprometer las posibilidades de curación y se ha observado que las dosis inferiores al tope propuesto han demostrado producir daño cardiaco subclínico ${ }^{3}$. Se ha propuesto también la administración de análogos antracíclicos, como la epirubicina, aunque se ha visto cardiotoxicidad grado 1 y $2^{3,31}$, o la administración de doxorubicina liposomal, que se asocia con bajas concentraciones de doxorubicina libre, con distribución limitada en el miocardio ${ }^{3}$. Se debe considerar el uso de dexrazoxano, agente quelante de hierro que inhibe la peroxidación de las membranas lipídicas con disminución de la cardiotoxicidad secundaria a antraciclinas ${ }^{27}$. La limitación de la dosis de radiación en el área cardiaca es también esencial para reducir las secuelas y complicaciones de la irradiación torácica a largo plazo. La dosis actualmente recomendada en niños y adolescentes tratados para la enfermedad de Hodgkin después de dos ciclos de quimioterapia es de 20 Gy (frente a 30 Gy de protocolos previos) ${ }^{60}$.

\section{Tratamiento}

Los fármacos frecuentemente estudiados y utilizados para tratar la cardiotoxicidad son los inhibidores de la enzima convertidora de la angiotensina (IECA), sin embargo los efectos beneficiosos de estos fármacos y su capacidad para reducir la morbimortalidad asociada a la antraciclina en los supervivientes de cáncer infantil parece ser transitoria ${ }^{36}$. El carvedilol proporciona cardioprotección mediante la inhibición de especies reactivas de oxígeno, la eliminación de radicales libres, la prevención de la peroxidación lipídica y el aumento de las concentraciones de vitamina $\mathrm{E}^{42}$. La insuficiencia cardiaca aguda y crónica deben ser tratadas de acuerdo con el momento de su presentación.

\section{Recomendaciones de evaluación y seguimiento cardiovascular}

No existe un consenso internacional actual de seguimiento de los pacientes pediátricos sometidos a tratamientos oncohematológicos. Algunas guías realizadas por grupos de expertos recomiendan la evaluación de pacientes pediátricos previa a la quimioterapia mediante la valoración de la función sistólica y diastólica del VI (FEVI inicial permisible $>50 \%$ ). Se recomienda otra evaluación después de la mitad de la dosis acumulativa total prescrita en el protocolo de tratamiento o al alcanzar $200 \mathrm{mg} / \mathrm{m}^{2}$ de doxorubicina, luego cada $100 \mathrm{mg} / \mathrm{m}^{2}$ en adelante. En el caso de pacientes con una FEVI inicial $<30 \%$, se debe estudiar la relación entre riesgo y beneficio respecto al tratamiento antitumoral, desaconsejándose el uso de fármacos cardiotóxicos, 


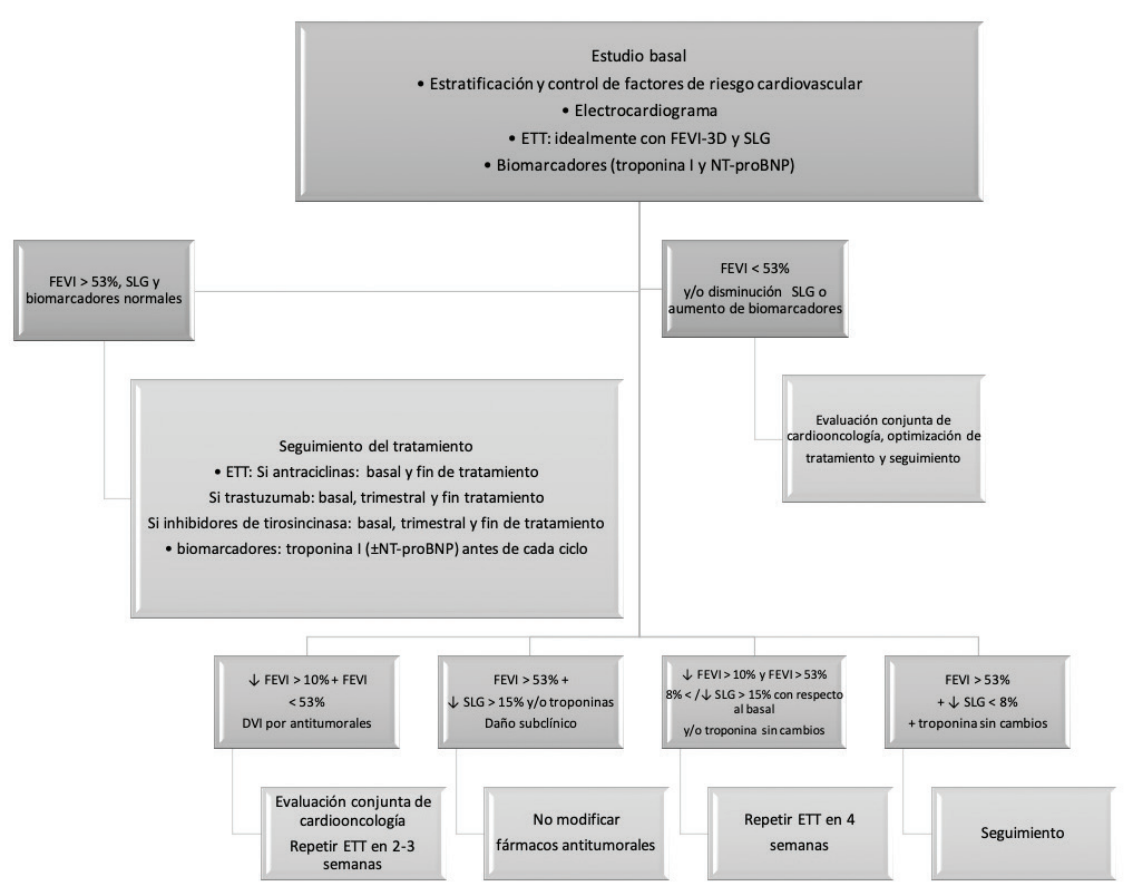

Figura 1. Algoritmo de evaluación del tratamiento de pacientes sometidos a fármacos antitumorales. ETT: ecocardiograma transtorácico; FEVI: fracción de eyección de ventrículo izquierdo; SLG: strain longitudinal global; NT-proBNP: fracción amino terminal del propéptido natriurético cerebral; DVI: disfunción ventricular izquierda.

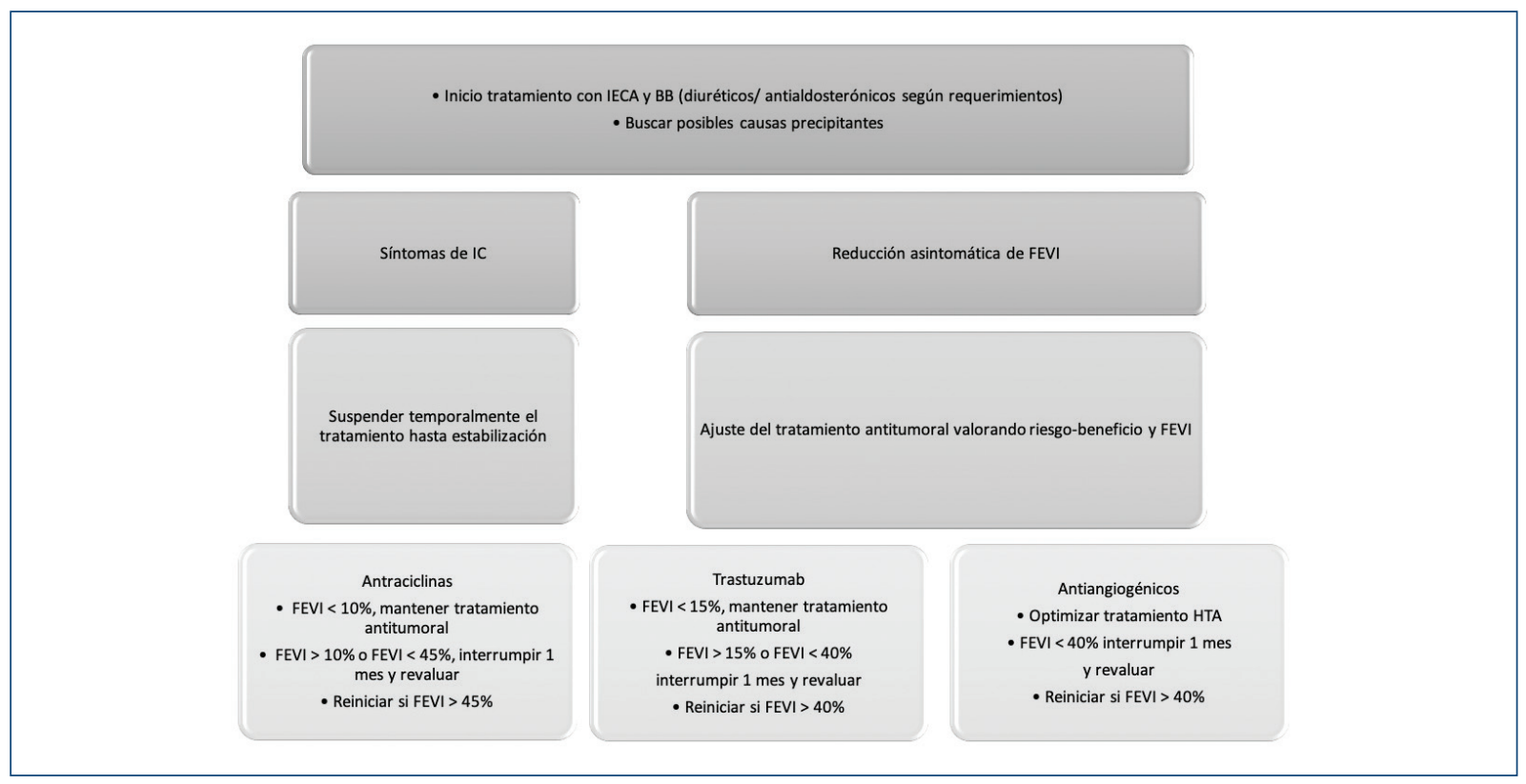

Figura 2. Algoritmo de evaluación y tratamiento de pacientes sometidos a fármacos antitumorales con FEVI disminuida. FEVI: fracción de eyección de ventrículo izquierdo; BB: betabloqueadores; IC: insuficiencia cardiaca; HTA: hipertensión arterial; IECA: inhibidores de la enzima convertidora de angiotensina. 


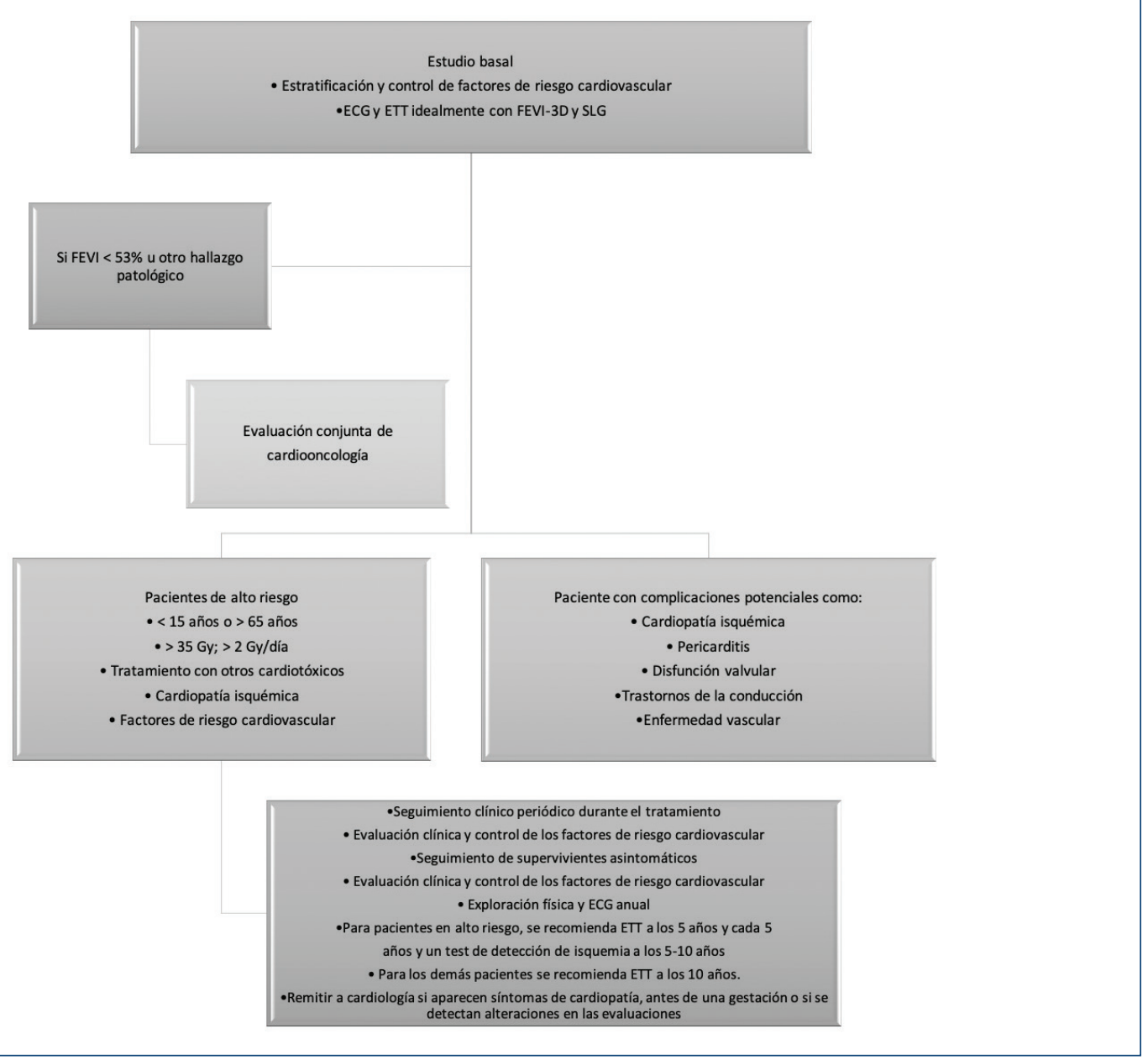

Figura 3. Algoritmo de seguimiento de pacientes sometidos a tratamiento con radioterapia que incluya total o parcialmente el corazón.

ETT: ecocardiograma transtorácico; FEVI: fracción de eyección de ventrículo izquierdo; SLG: strain longitudinal global.

especialmente antraciclinas. En pacientes con una FEVI basal entre el 30 y el 50\%, la evaluación ecocardiográfica debe realizarse antes de cada ciclo de quimioterapia ${ }^{12}$. Los pacientes que reciben dosis más altas de antraciclinas y los que reciben terapias combinadas deben contar con evaluaciones más frecuente de la función sistólica. En los pacientes con evidencia ecocardiográfica de enfermedad valvular y ante el antecedente de catéteres centrales, se debe realizar evaluación y profilaxis para la endocarditis bacteriana ${ }^{61}$. Se deben descartar la estenosis de la arteria carótida y subclavia (a los 10 años de recibida la radioterapia) en pacientes tratados con campos de radiación, incluyendo estos vasos a dosis de $40 \mathrm{~Gy}^{61}$.

En el documento de consenso y recomendaciones publicado por López Fernández, et al. en España en el año 2017 se marcan algunos lineamientos para el seguimiento inicial y a largo plazo de los pacientes sometidos a terapias antitumorales durante el tratamiento, en casos de disminución de la FEVI y en aquellos sometidos a radioterapia ${ }^{8}$ (Figs. 1-3). Si bien no son específicamente dirigidas a pacientes en edad pediátrica, se pueden considerar como referencia en la práctica, siempre considerando para las evaluaciones el estado clínico del paciente de forma individualizada.

\section{Equipos multidisciplinarios en cardio- onco-hematología}

Los equipos de cardio-onco-hematología reúnen a los profesionales implicados en la atención de pacientes con cáncer, con el objetivo de facilitar el tratamiento y minimizar la toxicidad cardiovascular. El desarrollo de 
protocolos de prevención y tratamiento precoz de la cardiotoxicidad evitará la suspensión precoz de los antitumorales, y permitirá optimizar los resultados en salud y reducir los costos ${ }^{8}$.

\section{Discusión}

El diagnóstico temprano del cáncer y la eficacia en su tratamiento ha llevado actualmente a una disminución de la mortalidad, sin embargo, también a complicaciones cardiovasculares reversibles e irreversibles; incluso, en algunos casos, el riesgo de muerte supera al ocasionado por recaídas tumorales ${ }^{62}$, motivos por los que es importante la identificación de factores de riesgo y un estrecho seguimiento por el cardiólogo, el oncólogo o el hematólogo mediante la interacción entre cada uno de ellos, para realizar una monitorización adecuada de cada paciente. Aunque existen algunas recomendaciones de grupos de expertos para niños y varias más en forma para adultos, es posible basarse en las que mejor se adapten a las características del enfermo y del equipo multidisciplinario. Así, se puede citar, además de los esquemas de seguimiento mencionados previamente, el publicado por Plana, et al. en 2014, en el que para el caso del tratamiento con antraciclinas, se recomienda una evaluación ecocardiográfica basal, con nueva evaluación al finalizar el tratamiento y después de seis meses. Si la dosis usada es $>240 \mathrm{mg} / \mathrm{m}^{2}$, se recomienda una evaluación en cada ciclo adicional de tratamiento ${ }^{11}$. Es preciso recordar que la principal estrategia para minimizar la cardiotoxicidad es su detección precoz, para implementar tempranamente un tratamiento preventivo de la disfunción ventricular ${ }^{50}$.

\section{Conclusiones}

Existe un número importante de padecimientos oncohematológicos en niños con diversas terapias antitumorales, que por su naturaleza afectan al sistema cardiovascular en su totalidad, por lo cual es necesario que cardiólogos pediatras, oncólogos y hematólogos tengan conocimiento de los potenciales efectos de los fármacos y de los factores de riesgo, así como sobre la prevención primaria y secundaria de cardiotoxicidad. Se recomienda la formación de equipos de trabajo multidisciplinario con los recursos humanos y materiales necesarios (clínicas de cardio-onco-hematología), acciones que a largo plazo se reflejarán en los costos hospitalarios y en un impacto positivo físico y emocional del paciente y su familia, así como en la mejora de la calidad integral de la atención.

\section{Financiamiento}

La presente investigación no ha recibido ninguna beca específica de agencias de los sectores público, comercial o sin ánimo de lucro.

\section{Conflicto de intereses}

Los autores declaran no tener conflicto de intereses.

\section{Responsabilidades éticas}

Protección de personas y animales. El autor declara que para esta investigación no se han realizado experimentos en seres humanos ni en animales.

Confidencialidad de los datos. El autor declara que han seguido los protocolos de su centro de trabajo sobre la publicación de datos de pacientes.

Derecho a la privacidad y consentimiento informado. El autor declara que en este artículo no aparecen datos de pacientes.

\section{Bibliografía}

1. Lipshultz SE, Diamond MB, Franco VI, Aggarwal S, Leger K, Santos MV, et al. Managing chemotherapy- related cardiotoxicity in survivors of childhood cancers. Paediatr Drugs. 2014;16(5):373-89.

2. Vargas-Neri JL, Castelán-Martínez OD, de Jesús Estrada-Loza M, Betanzos-Cabrera Y, Rivas-Ruiz R. Anthracycline-induced cardiotoxicity: report of fatal cases. Rev Med Inst Mex Seguro Soc. 2016;54(3):404-8.

3. Gallegos CS. Estrategias de cardioprotección en oncohematología pediátrica. GAMO. 2009;8(3):35-9.

4. Lipshultz SE, Colan SD, Gelber RD, Perez-Atayde AR, Sallan SE, Sanders SP. Late cardiac effects of doxorubicin therapy for acute lymphoblastic leukemia in childhood. N Engl J Med. 1991;324(12):808-15.

5. Kremer LCM, van Dalen EC, Offringa M, Voute PA. Frequency and risk factors of anthracycline-induced clinical heart failure in children: A systematic review. Ann Oncol. 2002:13(4):503-12

6. Mertens AC, Liu Q, Neglia JP, Wasilewski K, Leisenring W, Armstrong GT, et al. Cause-specific late mortality among 5 -year survivors of childhood cancer: The Childhood Cancer Survivor Study. J Natl Cancer Inst. 2008;100(19):1368-79.

7. Tukenova M, Guibout C, Oberlin O, Doyon F, Mousannif A, Haddy N, et al. Role of cancer treatment in long-term overall and cardiovascular mortality after childhood cancer. J Clin Oncol. 2010;28(8):1308-15.

8. López-Fernández T, Martín García A, Santaballa Beltrán A, Montero Luis Á, García Sanz R, Mazón Ramos P, et al. Cardio-onco-hematología en la práctica clínica. Documento de consenso y recomendaciones. Rev Esp Cardiol. 2017;70(6):474-86.

9. Zylberman M, Agüero R. Consenso de diagnóstico, prevención y tratamiento de la cardiotoxicidad por tratamiento médico del cáncer. Rev Argent Cardiol. 2013;81(5):1-64.

10. Albini A, Pennesi G, Donatelli F, Cammarota R, De Flora S, Noonan DM. Cardiotoxicity of anticancer drugs: the need for cardio-oncology and cardio-oncological prevention. J Natl Cancer Inst. 2010;102(1):14-25.

11. Plana JC, Galderisi M, Barac A, Ewer MS, Ky B, Scherrer-Crosbie M, et al. Expert consensus for multimodality imaging evaluation of adult patients during and after cancer therapy: a report from the ASE and the EACVI. J Am Soc Echocardiogr. 2014;27(9):911-39.

12. Santos MV, Paiva MG, Macedo CR, Petrilli AS, Azeka E, Jatene IB, et al.; Sociedade Brasileira de Cardiologia. I Diretriz brasileira de cardio-oncologia pediátrica da Sociedade Brasileira de Cardiologia. Arq Bras Cardiol. 2013;100(5 Supl.1):1-68.

13. Trachtenberg BH, Landy DC, Franco VI, Henkel JM, Pearson EJ, Miller TL, et al. Anthracycline-associated cardiotoxicity in survivors of childhood cancer. Pediatr Cardiol. 2011;32(3):342-53

14. Adams MJ, Lipshultz SE. Pathophysiology of anthracycline and radiation-associated cardiomyopathies: implications for screening and prevention. Pediatr Blood Cancer. 2005;44(7):600-6. 
15. Gilladoga AC, Manuel C, Tan CT, Wollner N, Sternberg SS, Murphy ML. The cardiotoxicity of adriamycin and daunomycin in children. Cancer. 1976;37(2 Suppl):1070-8.

16. Little MP, Azizova TV, Bazyka D, Bouffler SD, Cardis E, Chekin S, et al. Systematic review and meta-analysis of circulatory disease from exposure to low-level ionizing radiation and estimates of potential population mortality risks. Environ Health Perspect. 2012;120(11):1503-11.

17. Adams MJ, Lipsitz SR, Colan SD, Tarbell NJ, Treves ST, Diller L, et al. Cardiovascular status in long-term survivors of Hodgkin's disease treated with chest radiotherapy. J Clin Oncol. 2004;22(15):3139-48.

18. Landy DC, Miller TL, Lipsitz SR, Lopez-Mitnik G, Hinkle AS, Constine LS, et al. Cranial irradiation as an additional risk factor for anthracycline cardiotoxicity in childhood cancer survivors: an analysis from the cardiac risk factors in childhood cancer survivors study. Pediatr Cardiol. 2013;34(4):826-34

19. Lipshultz SE, Landy DC, Lopez-Mitnik G, Lipsitz SR, Hinkle AS, Constine LS et al. Cardiovascular status of childhood cancer survivors exposed and unexposed to cardiotoxic therapy. J Clin Oncol. 2012;30(10):1050-7.

20. Lipshultz SE, Lipsitz SR, Sallan SE, Dalton VM, Mone SM, Gelber RD, et al. Chronic progressive cardiac dysfunction years after doxorubicin therapy for childhood acute lymphoblastic leukemia. J Clin Oncol. 2005;23(12):2629-36.

21. Swain SM, Whaley FS, Ewer MS. Congestive heart failure in patients treated with doxorubicin: a retrospective analysis of three trials. Cancer. 2003;97(11):2869-79.

22. Lipshultz SE, Lipsitz SR, Mone SM, Goorin AM, Sallan SE, Sanders SP et al. Female sex and drug dose as risk factors for late cardiotoxic effects of doxorubicin therapy for childhood cancer. $N$ Engl $J$ Med. 1995;332(26):1738-43

23. Lipshultz SE, Scully RE, Lipsitz SR, Sallan SE, Silverman LB, Miller TL, et al. Assessment of dexrazoxane as a cardioprotectant in doxorubicin-treated children with high-risk acute lymphoblastic leukaemia: long term follow-up of a prospective, randomised, multicentre trial. Lancet Oncol. 2010;11(10):950-61.

24. Lipshultz SE, Rifai N, Dalton VM, Levy DE, Silverman LB, Lipsitz SR, et al. The effect of dexrazoxane on myocardial injury in doxorubicin-treated children with acute lymphoblastic leukemia. N Engl J Med. 2004;351(2):145-53.

25. Lipshultz SE, Lipsitz SR, Kutok JL, Miller TL, Colan SD, Neuberg DS, et al. Impact of hemochromatosis gene mutations on cardiac status in doxorubicin-treated survivors of childhood high-risk leukemia. Cancer. 2013:119(19):3555-62

26. Krischer JP, Epstein S, Cuthbertson DD, Goorin AM, Epstein ML, Lipshultz SE. Clinical cardiotoxicity following anthracycline treatment for childhood cancer: the Pediatric Oncology Group experience. J Clin Oncol. 1997; 15(4):1544-52

27. Velásquez CA, González M, Berrouet MC, Jaramillo N. Cardiotoxicidad inducida por la quimioterapia desde las bases moleculares hasta la perspectiva clínica. Rev Colomb Cardiol. 2016;23(2):104-11.

28. Florescu M, Cinteza M, Vinereanu D. Chemotherapy-induced cardiotoxicity. Mædica. 2013;8(1):59-67

29. Dolci A, Dominici R, Cardinale D, Sandri MT, Panteghini M. Biochemical markers for prediction of chemotherapy-induced cardiotoxicity: systematic review of the literature and recommendations for use. Am J Clin Pathol. 2008;130(5):688-95.

30. Frishman WH, Sung HM, Yee HC, Liu LL, Keefe D, Einzig Al, et al. Cardiovascular toxicity with cancer chemotherapy. Curr Probl Cancer. 1997;21(6):301-60.

31. Pai VB, Nahata MC. Cardiotoxicity of chemotherapeutic agents: incidence, treatment and prevention. Drug Saf. 2000;22(4):263-302.

32. Rang HP. Rang et Dale's pharmacology. Edinburgh: Elsevier, Churchill Livingstone; 2016.

33. Hurtado S, Mejía AM, Sanabria A. Cardiotoxicidad por quimioterapia. Un enfoque práctico para el clínico. Insufic Card. 2011;6(3):131-43.

34. Adão R, de Keulenaer G, Leite-Moreira A, Brás-Silva C. Cardiotoxicidade associada à terapêutica oncológica: mecanismos fisiopatológicos e estratégias de prevencão. Rev Port Cardiol. 2013;32(5):395-409.

35. Slørdal L, Spigset O. Heart failure induced by non-cardiac drugs. Drug Saf. 2006;29(7):567-86

36. Lipshultz SE, Cochran TR, Franco VI, Miller TL. Treatment-related cardiotoxicity in survivors of childhood cancer. Nat Rev Clin Oncol. 2013; 10(12):697-710

37. Kremer LC, van Dalen EC, Offringa M, Ottenkamp J, Voûte PA. Anthracycline-induced clinical heart failure in a cohort of 607 children: long-term follow-up study. J Clin Oncol. 2001;19(1):191-6.

38. Bagnes C,Panchuk PN, Recondo G. Antineoplastic chemotherapy induced QTc prolongation. Curr Drug Saf. 2010;5(1):93-6.

39. Serrano C, Cortés J, De Mattos-Arruda L, Bellet M, Gómez P, Saura C, et al. Trastuzumab-related cardiotoxicity in the elderly: a role for cardiovascular risk factors. Ann Oncol. 2012;23(4):897-902.

40. Chavez-MacGregor M, Zhang N, Buchholz TA, Zhang Y, Niu J, Elting L, et al. Trastuzumab-related cardiotoxicity among older patients with breast cancer. J Clin Oncol. 2013;31(33):4222-8.
41. Lipshultz SE, Sambatakos P, Maguire M, Karnik R, Ross SW, et al Cardiotoxicity and cardioprotection in childhood cancer. Acta Haematol. 2014;132(3-4):391-9.

42. Diamond M, Franco V. Preventing and treating anthracycline-related cardiotoxicity in survivors of childhood cancer. Curr Cancer Ther Rev. 2012;8:141-51.

43. Dillenburg RF, Nathan $P$, Mertens L. Educational paper: decreasing the burden of cardiovascular disease in childhood cancer survivors: an update for the pediatrician. Eur J Pediatr. 2013;172(9):1149-60.

44. Zamorano JL, Lancellotti P, Muñoz DR, Aboyans V, Asteggiano R, et al. Position Paper on cancer treatments and cardiovascular toxicity developed under the auspices of the ESC Committee for Practice Guidelines: The Task Force for cancer treatments and cardiovascular toxicity of the European Society of Cardiology (ESC). European Heart J. 2016;37(36):2768-801.

45. Di Lisi D, Bonura F, Macaione F, Peritore A, Meschisi M, Cuttitta F, et al. Chemotherapy-induced cardiotoxicity: role of the tissue Doppler in the early diagnosis of left ventricular dysfunction. Anticancer Drugs. 2011;22(5):468-72

46. Nagueh SF, Middleton KJ, Kopelen HA, Zoghbi WA, Quinones MA. Doppler tissue imaging: a noninvasive technique for evaluation of left ventricular relaxation and estimation of filling pressures. J Am Coll Cardiol. 1997;30(6):1527-33

47. Sawaya H, Plana JC, Scherrer-Crosbie M. Newest echocardiographic techniques for the detection of cardiotoxicity and heart failure during chemotherapy. Heart Fail Clin. 2011;7(3):313-21.

48. Sawaya H, Sebag IA, Plana JC, Januzzi JL, Ky B, Cohen V, et al. Early detection and prediction of cardiotoxicity in chemotherapy-treated patients. Am J Cardiol. 2011;107(9):1375-80.

49. Plana JC, Galderisi M, Barac A, Ewer MS, Ky B, Scherrer-Crosbie M, et al. Expert consensus for multimodality imaging evaluation of adult patients during and after cancer therapy: A Report from the American Society of Echocardiography and the European Association of Cardiovascular Imaging. J Am Soc Echocardiogr. 2014;27(9):911-39.

50. Cardinale D, Colombo A, Lamantia G, Colombo N, Civelli M, De Giacomi G et al. Anthracycline-induced cardiomyopathy: clinical relevance and response to pharmacologic therapy. J Am Coll Cardiol. 2010;55(3):213-20.

51. Alpert JS, Thygesen K, Antman E, Bassand JP. Myocardial infarction redefined a consensus document of The Joint European Society of Cardiology/American College of Cardiology Committee for the redefinition of myocardial infarction. J Am Coll Cardiol. 2000;36(3):959-69.

52. Cardinale D, Sandri MT, Colombo A, Colombo N, Boeri M, Lamantia G, et al. Prognostic value of troponin I in cardiac risk stratification of cancer patients undergoing high-dose chemotherapy. Circulation. 2004;109(22):2749-54

53. Nousiainen T, Vanninen E, Jantunen E, Puustinen J, Remes J, Rantala A, et al. Natriuretic peptides during the development of doxorubicin-induced left ventricular diastolic dysfunction. J Intern Med. 2002;251(3):228-34.

54. Cardinale D, Colombo A, Torrisi R, Sandri MT, Civelli M, Salvatici M, et al. Trastuzumab-induced cardiotoxicity: clinical and prognostic implications of troponin I evaluation. J Clin Oncol. 2010;28(25):3910-6.

55. Januzzi JL Jr, Rehman SU, Mohammed AA, Bhardwaj A, Barajas L, Barajas $\mathrm{J}$, et al. Use of amino-terminal pro-B-type natriuretic peptide to guide outpatient therapy of patients with chronic left ventricular systolic dysfunction. J Am Coll Cardiol. 2011;58(18):1881-9.

56. Masson S, Latini R, Anand IS, Barlera S, Angelici L, Vago T, et al. Prognostic value of changes in $\mathrm{N}$-terminal pro-brain natriuretic peptide in Val-HeFT (Valsartan Heart Failure Trial). J Am Coll Cardiol. 2008(12); 52:997-1003.

57. Sandri MT, Salvatici M, Cardinale D, Zorzino L, Passerini R, Lentati $P$, et al. N-terminal pro-B-type natriuretic peptide after high-dose chemotherapy: a marker predictive of cardiac dysfunction? Clin Chem. 2005; 51(8):1405-10

58. Cil T, Kaplan AM, Altintas A, Akin AM, Alan S, Isikdogan A. Use of $\mathrm{N}$-terminal pro-brain natriuretic peptide to assess left ventricular function after adjuvant doxorubicin therapy in early breast cancer patients: a prospective series. Clin Drug Investig. 2009;29(2):131-7.

59. Fresneau B, Fayech C, Butel T, Haddy N, Valteau-Couanet D, Ou P. Facteurs de risque et surveillance à long terme des complications cardiaques après traitement pour un cancer pendant l'enfance. Rev Med Interne. 2017;38(2):125-32.

60. Shankar SM, Marina N, Hudson MM, Hodgson DC, Adams MJ, Landier W et al. Monitoring for cardiovascular disease in survivors of childhood cancer: Report from the Cardiovascular Disease Task Force of the Children's Oncology Group. Pediatrics. 2008;121(2):e387-96.

61. Witteles RM, Bosch X. Myocardial protection during cardiotoxic chemotherapy. Circulation. 2015;132(19):1835-45.

62. Nhola LF, Villarraga HR. Rationale for Cardio-Oncology Units. Rev Esp Cardiol. 2017;70(7):583-89. 